\title{
L'ESPERIENZA DEL DOLORE TRA FILOSOFIA E NEUROSCIENZE
}

\author{
Nota del s.c. LUCA VANZAGO (*)
}

(Adunanza dell'11 marzo 2021)

SuNTO. - In questo saggio mi propongo di analizzare due posizioni fondamentali emerse recentemente nel dibattito filosofico in relazione all'esperienza del dolore: quello riduzionistico di area neurocognitiva e quello fenomenologico. La comparazione è condotta alla luce della nozione di rappresentazione, per mostrare che la prospettiva riduzionistica non riesce a dar conto in maniera soddisfacente dell'aspetto propriamente esperienziale del dolore, che invece l'approccio fenomenologico riesce a cogliere più adeguatamente.

$* * *$

ABSTRACT. - In this essay I propose to analyze two fundamental positions that have recently emerged in the philosophical debate in relation to the experience of pain: the reductionist one peculiar to the neurocognitive area, and the phenomenological one. The comparison is conducted in the light of the notion of representation, in order to show that the reductionist perspective fails to give a satisfactory account of the properly experiential aspect of pain, which instead the phenomenological approach is able to grasp more adequately.

Analisi e riflessioni sul complesso fenomeno chiamato dolore sono antiche quanto l'uomo e certamente presenti fin dall'inizio della storia del pensiero filosofico, tuttavia si può dire che recentemente questo tema stia

(") Dipartimento di Studi umanistici, Università di Pavia, Italy.

E-mail: luca.vanzago@unipv.it 
tornando alla ribalta dell'attenzione degli studiosi, non soltanto dei filosofi ma, anche e forse soprattutto, dei medici, degli scienziati cognitivi, dei biologi, e insomma di ricercatori che operano in ambiti solitamente meno direttamente coinvolti in questioni che potremmo, almeno provvisoriamente, chiamare soggettive o per altro verso, qualitative.

In realtà il problema di come comprendere il dolore è da tempo presente anche nella riflessione epistemologica sulla medicina, e anzi forse il ritorno di interesse per questo aspetto universale ma certo poco piacevole dell'esistenza umana può essere motivato innanzi tutto dalla crescente preoccupazione dei medici per un aspetto della sofferenza che, da appendice inevitabile della malattia, si sta progressivamente trasformando in patologia autonoma, soprattutto in connessione con l'invecchiamento della popolazione. La International Association for the Study of Pain, che pubblica l'importante rivista internazionale Pain, è stata fondata nel 1973 e ora vede al proprio interno più di 7000 membri provenienti da 133 nazioni, ha 90 sedi nazionali, e 20 gruppi di interesse speciale, dedicati ad altrettanti temi rilevanti connessi alla ricerca generale sul dolore.

Chiaramente dunque la questione della comprensione del dolore riapre un capitolo del più generale dibattito su cosa sia l'esperienza, e in particolare se la coscienza abbia un ruolo oppure no. Ma va tenuto in conto anche l'aspetto della comunicazione, non necessariamente linguistica, dell'esperienza dolorosa. In effetti vi sono questionari per la valutazione del dolore che propongono un esame delle espressioni facciali o dei comportamenti gestuali, quando non sia ad es. possibile ottenere uno scambio verbale significativo con la persona sofferente. In questo senso il corpo "parla" anche se non utilizza il linguaggio.

Questi due problemi sono chiaramente interconnessi e aprono a un dibattito epistemologico che chiama in causa la più generale problematica relativa a cosa sia l'esperienza, e in particolare come si debba comprendere l'aspetto fenomenico, in questo caso connesso alla sofferenza, rispetto a un più o meno chiaramente concepito sostrato corporeo o materiale. In definitiva l'esperienza del dolore riapre il vaso di pandora del contrasto tra dualismo e monismo e dunque la grande questione del rapporto tra mente e corpo. Forse però l'indagine sul dolore può offrire uno sguardo diverso su questi temi tradizionali. In quanto segue cercherò di schematizzare due tra i modelli concettuali prevalenti senza peraltro poter neppure presumere di esaurire tutti gli aspetti impliciti in questo problema. 


\section{LE PROSPETTIVE RIDUZIONISTICHE}

Tra le opere di coloro che a vario titolo possono essere compresi in questo tipo di approccio si possono annoverare in particolare quelle dei coniugi Churchland e di Daniel Dennett. Le loro posizioni sono molto note e apprezzate tra gli studiosi di neuroscienze. Ho scelto per ragioni di economia di spazio di concentrarmi su di un saggio dei coniugi Paul e Patricia Churchland. ${ }^{2}$

L'esordio del saggio Neurofilosofia, con cui i Churchland esprimono in modo sintetico e come in un manifesto programmatico la propria posizione, è esemplare nel chiarire anche la loro concezione del dolore:

Gli sviluppi conoscitivi delle basi neurobiologiche della percezione, dell'attenzione, della memoria, dell'apprendimento, del linguaggio, del sonno e della pianificazione delle azioni prefigurano per le scienze cognitive e le neuroscienze la possibilità di spiegare a livello di attività cerebrale anche la coscienza e la qualità delle esperienze soggettive. Che cosa ne sarà delle definizioni e dei concetti psicologici una volta che le relative funzioni saranno state ricondotte alle proprietà di specifiche reti di neuroni o meccanismi neurobiologici? Si tratta di una questione non banale, perché implica un giudizio di validità per quanto riguarda gli studi psicologici e di realtà in merito ai fenomeni descritti dalla psicologia. Nell'ambito delle neuroscienze l'atteggiamento epistemologico prevalente è il materialismo eliminativo, per cui concetti quali credenza, libero arbitrio e coscienza potranno e dovranno essere ridefiniti e spiegati via via che la ricerca empirica farà luce sulla natura delle funzioni cerebrali.

L'approccio riduzionista basato sull'eliminazione sostiene che la spiegazione di un macrofenomeno nei termini delle sue dinamiche a livello microstrutturale non significa che il macrofenomeno stesso, come per esempio il dolore, non sia reale, né che rappresenti qualcosa di ridondante che non vale la pena di spiegare scientificamente. La strategia riduzionista implica non una spiegazione diretta dei fenomeni che accadono ai livelli superiori in termini di fenomeni che interessano $\mathrm{i}$ livelli organizzativi inferiori, ma progressive spiegazioni riduttive che comunque prevedono che la ricerca proceda simultaneamente a tutti $\mathrm{i}$ livelli.

1 Il saggio è pubblicato online in Enciclopedia della scienza e della tecnica Treccani al sito: http://www.treccani.it/enciclopedia/neurofilosofia_\%28Enciclopediadella-Scienza-e-della-Tecnica\%29/ 
Il materialismo eliminativo implica sul piano metodologico un approccio naturalistico, in quanto assume che nessun metodo a priori possa da solo far scoprire la natura della mente, e che sia possibile spiegare in termini di attività nervosa, di evoluzione del cervello e di interazione tra cervello e cultura anche scelte e responsabilità morali. Tale teoria accetta una nozione non mistica di emergenza, intesa come proprietà di una rete nervosa dovuta alle proprietà intrinseche dei neuroni e al loro modo di interagire.

Una proprietà emergente è una proprietà scientificamente spiegabile, non una proprietà non fisica non spiegabile dalla scienza. I sistemi nervosi presentano molti livelli di organizzazione, i quali spaziano dalla scala molecolare a quella di complessi funzionali non cellulari, come le spine dendritiche, ai neuroni, alle piccole e grandi reti, alle aree, fino ai sistemi. Sebbene si debba sempre stabilire in modo empirico quali siano i livelli funzionalmente significativi, risulta improbabile che $\mathrm{i}$ macroeffetti dovuti all'attività di un apparato funzionale, come per esempio la percezione del movimento, possano essere spiegati richiamando direttamente le proprietà dei livelli di organizzazione più elementari. Più verosimilmente, le prestazioni dei network di livello più elevato saranno l'effetto dell'attività dei network più piccoli, i quali a loro volta saranno l'effetto dei neuroni che vi partecipano e delle loro interconnessioni, che a loro volta dipenderanno per le loro attività dalle proprietà dei canali proteici, da quelle dei neuromodulatori e dei neurotrasmettitori.

I Churchland sono consapevoli del fatto che tale impostazione suscita un'obiezione di fondo, ossia quella fondata sulla specificità dell'esperienza qualitativa di fenomeni che, come il dolore in particolare, non possono essere risolti in una prospettiva in terza persona e causale. Come essi stessi sostengono, le argomentazioni contro il materialismo si basano su quanto segue. Il carattere qualitativo intrinseco (ossia il "quale") di una sensazione deve essere nettamente distinto dai numerosi aspetti causali, funzionali e relazionali che lo caratterizzano. Riprendiamo l'esempio del dolore: esso è il risultato di un danno subito dall'organismo e provoca nell'individuo uno stato di infelicità e una reazione di evitamento. L'esigenza filosofica riduzionista è quella di individuare gli stati del cervello che esprimono il medesimo profilo causale e relazionale precedentemente accettato da noi come caratteristico dello stato di dolore. Se nel confronto tra questi stati del cervello che esprimono lo stesso profilo causale si rinvenissero caratteri sistematici, potremmo legittimamente affermare di avere scoperto in che cosa consiste il dolore, ovvero in uno stato cerebrale. 
Nel sostenere tale tesi dovremmo confrontarci però con una difficoltà: il quale intrinseco del dolore dovrebbe essere ignorato dall'analisi, come lo sarebbe la conoscenza intima che ogni individuo ha dei qualia del proprio dolore. Si tratta di un problema di difficile risoluzione, perché il dato dell'autocoscienza non si esaurisce nella struttura di rapporti causali o di altra natura entro cui si colloca. Mentre in tutti gli altri ambiti della scienza il problema della natura essenziale dei fenomeni è affrontato in termini riduzionistici, nel caso unico dell'autocoscienza ci troviamo di fronte a un insieme di proprietà intrinseche (il colore legato a un ricordo, il profumo di una rosa e tutti gli altri qualia soggettivi che animano la nostra vita interiore) la cui essenza non può essere colta con argomenti di natura causale, funzionale, strutturale o relazionale. In altre parole, secondo le tesi antiriduzionistiche, i qualia soggettivi delle sensazioni costituiscono un qualcosa di aggiuntivo e di diverso rispetto al ruolo causale e relazionale che le sensazioni stesse svolgono nell'economia biologica e cognitiva individuale. Per quanto le nostre capacità introspettive innate ci consentano di valutare, tali sensazioni correlate al quale, per esempio di un dolore o del colore rosso, non rivelano in alcun modo elementi costitutivi o una struttura relazionale. Possiamo riconoscerle e distinguerle in modo spontaneo, certo e inequivocabile, ma non siamo in grado di dire come o su quali basi. Di conseguenza, i qualia sensoriali appaiono come delle entità intrinsecamente e assolutamente semplici; inoltre, la nostra conoscenza di essi non sembra poter essere più diretta e fondata ed essi non sembrano dunque analizzabili dalle scienze fisiche.

In questo senso dunque i Churchland ritengono che si possa superare l'obiezione per cui il dolore conterrebbe un aspetto qualitativo irriducibile alla sua riconduzione all'analisi dei correlati neurali. Sulla base di questa modellizzazione, ritengono infatti che si possa giungere a ricondurre i cosiddetti "qualia", di cui il dolore è espressione eminente, a rappresentazioni che, a loro volta, possano essere inquadrate in un modello "scientifico": per quanto concerne il problema cruciale della coscienza, dunque, si potrebbe supporre che una rappresentazione entri a farne parte qualora il suo vettore di attivazione si trovi presso una particolare popolazione di cellule, all'interno di un adeguato sistema nervoso centrale ricorrente, che unifichi le numerose modalità sensoriali e che controlli il comportamento motorio. Che risponda o meno a verità, l'ipotesi riduzionistica che abbiamo formulato ci fornisce 
in primo luogo una possibile spiegazione fisica della natura 'intrinseca' dei qualia sensoriali, che altro non sarebbero che vettori di attivazione, e ci permette inoltre di esplorare la possibilità che tali vettori si trasformino in autocoscienza manifestandosi come parte dell'attività di rappresentazione di una rete adeguatamente strutturata.

La prospettiva qui delineata solleva una quantità di problemi che evidentemente non possono essere discussi in questa sede. Vorrei soffermarmi allora soltanto su di uno di essi, che tuttavia mi pare cruciale. I Churchland impostano la propria analisi all'interno di un quadro concettuale per cui i qualia possono, e in effetti devono, essere compresi in termini di rappresentazioni. A questo patto è poi possibile ricondurre la nozione di quale entro l'approccio oggettivante e retto da una modellizzazione sostanzialmente causalistica. $\mathrm{Ci}$ si può però chiedere se il dolore possa essere inteso in termini di rappresentazioni.

\section{LE PROSPETTIVE FENOMENOLOGICHE}

Si tratta, come noto, di una problematica estremamente vasta e diversificata. Considerando solo le analisi fenomenologiche in senso stretto, e quindi partendo dagli scritti di Husserl, si deve innanzi tutto notare come già il fondatore stesso della fenomenologia abbia progressivamente sviluppato posizioni diverse relativamente alla questione di come comprendere il fenomeno emotivo. Nelle Ricerche logiche Husserl segue un modello ereditato dal pensiero dominante all'epoca ed elaborato in particolare da C. Stumpf, il quale se da una parte introduce importanti innovazioni rispetto al fenomenismo empiristico della psicologia positivistica, intende comunque le emozioni in termini di strutture fondate su soggiacenti rappresentazioni cognitive. Husserl sostiene una tesi analoga quando afferma che "le intenzioni affettive (Gemütsintentionen) si basano su intenzioni rappresentazionali o giudicative" . Il ragionamento è piuttosto semplice: per poter provare emozioni rispetto a qualcosa, è necessario prima averlo percepito o rappresentato intenzionalmente. Ė altrettanto evidente che tale considerazione non può essere applicata al dolore, quanto meno al dolore fisico, in quanto esso si dà in maniera affatto svincolata da qualunque rappresen-

2 Husserl 1988, p. 159. 
tazione intenzionale, e anzi è proprio tale mancanza di oggetto (anche se non di correlazione, questione su cui tornerò) uno degli elementi strutturali della peculiare "logica" del dolore.

Già nel primo volume delle Idee, in ogni caso, Husserl apporta una trasformazione rilevante alla propria posizione, considerando le emozioni come atti intenzionali, in grado di far apparire oggetti di nuovo tipo. Le emozioni cioè non sono più mere colorazioni di atti cognitivi precedenti (in senso strutturale) e in quanto tali fondanti, ma al contrario vengono comprese nella loro capacità di mostrare aspetti dell'essere a cui gli atti cognitivi non accedono. Gli atti emotivi si strutturano secondo due assi fondamentali: consentono la presa di posizione affettiva verso il mondo, indipendentemente dagli atteggiamenti proposizionali, e permettono al soggetto di incontrare se stesso secondo determinate possibilità. Questo non significa che Husserl consideri l'affettività come una sorta di fusione emotiva, ma al contrario questa tematica lo porta ad attribuire capacità oggettivante agli stessi atti emotivi, considerando però appunto come "di nuova specie" gli oggetti così intenzionati.

Husserl distingue inoltre gli atti intenzionali emotivi dalle tonalità emotive, termine che si è soliti attribuire piuttosto a Heidegger, in quanto ritiene che con queste ultime ad apparire sia piuttosto un certo senso della totalità, e non quindi un determinato affetto, connesso a un individuo in senso fenomenologico. Una diversa tonalità emotiva non cambia una relazione affettiva a questo o quel determinato ente, quanto piuttosto produce una diversa modalità di manifestazione di un mondo, e quindi in definitiva un mondo diverso. La tonalità emotiva rappresenta quindi lo sfondo emozionale dell'apparire del senso di un mondo. In un manoscritto dei primi anni Venti, Husserl sostiene che lo sfondo affettivo non costituisce alcun oggetto particolare, dunque neppure gli oggetti di nuova specie di cui parla nelle Idee, quanto piuttosto esso è un "elemento unitario non chiaro, un essere in una tensione confusa e tuttavia priva di direzione" (Ms. A VI 26/3). Di questa determinazione va qui ritenuta soprattutto l'indicazione della tensione come elemento strutturale fondamentale.

È la tonalità emotiva a "colpire", propriamente, il soggetto, il quale allora si risveglia, diviene desto per il contenuto nella sua caratteristica emozionale (Ms. B III 9/18b). Sono le affezioni stesse dunque a venir incontrate all'interno di una peculiare tonalità, la quale condiziona ogni singola affezione e si propaga lungo ognuna di esse. A volte dunque si può dare il caso che una affezione che apparterrebbe a un 
determinato registro, ad es. una gioia relativa a eventi giocosi, non riesca a far breccia nel suo senso "oggettivo" in una esperienza personale condizionata, ad es., da una tonalità emotiva depressa o melanconica. Tale considerazione suggerisce pertanto due riflessioni. Innanzi tutto si può notare che la vita emotiva è costituita essenzialmente da strutture generali, le tonalità emotive, che ne determinano il livello di ricettività.

In secondo luogo, e propriamente, si può allora porre il problema dell'esperienza del dolore in termini di una sua determinazione strutturale fondamentale. Il dolore accade non a un corpo inerte o meccanico, che come tale nulla potrebbe neppure sapere relativamente alla sua condizione di sofferenza, ma a una struttura caratterizzata da una ricettività generale determinata in base alla propria "intonabilità" emotiva, se mi si passa l'espressione, ossia secondo una struttura trascendentale di stampo affettivo e non cognitivo, che costituisce propriamente la condizione di possibilità dell'esperire in generale e dell'esperire affettivo in particolare.

A partire da questa prima determinazione si può allora anche osservare che il dolore può correttamente essere compreso soltanto se lo si considera in termini dinamici. Esso infatti non "è" puramente e semplicemente, cioè non sussiste, ma accade e interviene, cosa che anche il senso comune del resto riconosce, nella misura in cui, all'interno di una struttura di esperienza affettiva determinata già sempre in un determinato modo, un evento può, ma può anche non, propagarsi.

Ma poi e soprattutto, un evento di natura affettiva peculiare, come è una esperienza dolorosa, si caratterizza per il suo rompere in maniera forzata e traumatica una tonalità emotiva, qualunque essa sia. Il dolore si caratterizza cioè anche per la sua dinamicità, quindi sia per il fatto di avere una natura temporalmente connotata, fosse anche di marca estemporanea e istantanea, sia per il fatto che un dolore non può essere compreso che se viene considerato come proprio di un soggetto vivente, ossia temporalmente atteggiato.

Naturalmente qui si apre il capitolo fondamentale della correlazione tra vita e temporalità, su cui non mi posso soffermare se non facendo osservare che forse il dolore costituisce una possibile porta per comprendere il nesso tra tempo e vita, e non una sua manifestazione derivata. In altre parole forse si può dire che il dolore è la marca strutturale del corpo vivente, e cioè di un corpo segnato intrinsecamente dall'apertura rispetto a se stesso, da intendersi come una non-chiusura che ne fa un ente dinamico in senso ontologico, ossia inaugurante un 
disequilibrio, che non è una determinazione accessoria ma l'essenza stessa del "vivere" in senso verbale.

In chiusura di questo mio intervento non posso che osservare che non si tratta certamente di concludere, poiché la ricerca è appena agli inizi. Si può dire però che alcuni risultati conseguiti da una prima ricognizione delle diverse modellizzazioni disponibili spinge a operare in direzione di una revisione del quadro concettuale pur già articolato e complesso che soggiace alla redazione della definizione di dolore data dalla IASP. Attraverso la cooperazione interdisciplinare con neuroscienziati, linguisti, psicologi, neurologi e fisiologi, la filosofia può introdurre alcune considerazioni fondamentali di carattere categoriale, atte a rivedere piuttosto significativamente il presupposto ontologico che regge l'approccio analitico per metterlo in questione non da un punto di vista speculativo, ma a partire da esigenze pratiche e si potrebbe dire empiriche. Forse è proprio da un empirismo radicale, come direbbe James, ma quindi un empirismo che metta in discussione anche l'ontologia soggiacente la prospettiva cognitivistica riduzionistica, che si può muovere in direzione di una ricomprensione del dolore nel suo manifestarsi che resti più aderente alla complessità del fenomeno.

\section{BIBLIOGRAFIA}

Husserl, Edmund 1988 Ricerche logiche, Milano, Il Saggiatore.

Churchland, Paul and Churchland, Patricia 2007, Neurofilosofia <url: http://www.treccani.it/enciclopedia/neurofilosofia_\%28Enciclopedia-della-Scienza-e-dellaTecnica $\% 29 />$ 
